\title{
V. Kozhevnikov: Thermodinamics of Magnetizing Materials and Superconductors
}

\author{
CRS Press, Taylor and Francis Group, 2020
}

\author{
Michail Raikh ${ }^{1}$
}

Received: 27 August 2019 / Accepted: 4 September 2019/Published online: 26 October 2019

(C) Springer Science+Business Media, LLC, part of Springer Nature 2019

The book of Professor Kozhevnikov covers an important chapter of thermodynamics, which is largely underrepresented in the literature. To the best of my knowledge, this is the first monograph which consistently expounds the concepts of thermodynamics of materials in magnetic fields. In particular, it comprehensively addresses an issue of a demagnetizing factor and the forms of thermodynamic potentials appropriate for different sample/field configurations. Significant part of the book is devoted to the superconductivity. It is distinguished in in-depth discussions of not well-covered subjects, such as the intermediate state in type-I superconductors and magnetic properties of type-II materials with non-zero demagnetizing factor. In the first chapter (Elements of magnetostatics in magnetizing media), the author discusses latest achievements in the studies of superconductivity made possible due to the most advanced methods of magnetometry, such as the muon spin rotation spectroscopy. These achieve- ments include (but not limited to) a novel explanation of nucleation of superconductivity at high magnetic field and direct measurements of the field intensity $\mathrm{H}$ in type-I superconductors.

The book is written in a clear language without mathematical excesses but with an emphasis on the physical meaning of the concepts covered. To illustrate these concepts, all chapters are accompanied by original problems with solutions.

This book will definitely appeal to students and instructors/ researchers in Physics, Applied Physics, Chemistry, Material Science, and Electrical Engineering Departments. It can be used as a supplementary text in variety of courses, e.g., thermodynamics, electromagnetism, physics of condensed matter, superconductivity, and statistical physics.

Publisher's Note Springer Nature remains neutral with regard to jurisdictional claims in published maps and institutional affiliations.
Michail Raikh

raikh@physics.utah.edu

1 University of Utah, Salt Lake City, USA 